\title{
Assoziationen zu muslimischen Patienten in der Hausarztpraxis - Eine Befragung deutscher Allgemeinmediziner
}

\section{Associations with Muslim Patients in General Practice Surgeries - A Survey among German General Practicioners}

Autoren
Institut

\author{
A. Kronenthaler, H. Hiltner, M. Eissler
}

Lehrbereich Allgemeinmedizin, Universitätsklinikum Tübingen, Tübingen

\section{Schlüsselwörter \\ - Muslimische Patienten \\ - Allgemeinmedizin \\ interkulturelle Handlungs- kompetenz \\ - Compliance \\ - Vorurteile \\ - Qualitative Forschung}

\section{Key words}

- muslim patients

- general practice

- cross-cultural communication

- compliance

- stereotypes

qualitative research

\section{Bibliografie}

DOI http://dx.doi.org/

10.1055/s-0034-1367007

Online-Publikation: 8.5.2014

Gesundheitswesen 2014;

76: 434-439

(c) Georg Thieme Verlag KG

Stuttgart · New York

ISSN 0941-3790

Korrespondenzadresse

Dr. Andrea Kronenthaler

Lehrbereich Allgemeinmedizin

Universitätsklinikum Tübingen

Österbergstraße 9

72074 Tübingen

andrea.kronenthaler@medizin. uni-tuebingen.de

\section{Zusammenfassung}

$\nabla$

Einleitung: Steigende Bevölkerungszahlen von Muslime in Deutschland ${ }^{1}$ - aktuell etwa 4,3 Millionen - bedingen ein zunehmend häufigeres Auftreten als Patienten in hausärztlichen Praxen. Dennoch sind ihre heterogenen kulturellen und religiösen Lebenshintergründe vielfach für die behandelnden Hausärzte unbekannt und fremdartig. Um von deren Erfahrungen ausgehend Entwicklungen der interkulturellen Handlungskompetenz zu erfassen, wurde in der vorliegenden Untersuchung ein Brainwriting mit Hausärzte durchgeführt, das ihre spontanen Assoziationen mit muslimischen Patienten erfasst.

Methodik: 90 Hausärzte (66 männlich, 24 weiblich) notierten subjektive Gedanken und Stichwörter zu „Muslimischer Patient“ und ohne Austausch auf ein vorbereitetes Blatt Papier. Auf diesem wurden zusätzlich Geschlecht, Alter, Anzahl der Jahre als niedergelassender Hausarzt und Häufigkeit der Behandlung von muslimischen Patienten in der eigenen Praxis abgefragt. Anhand der Notizen konnten mit dem Datenauswertungsprogramm MAXQDA folgende analytische Kategorien „Sprache“, „Untersuchung“, „Kopftuch“, „Männer/Frauen“, „Begleitung“, „Gewalt“, „Krankheitsverständnis“, „Psychossomatik“ und „Compliance“, herausgearbeitet werden. Diese wurden inhaltsanalytisch ausgewertet.

Ergebnisse: Die notierten Gedanken zeigen, dass viele befragte Hausärzte die Behandlung muslimischer Patienten als schwierig wahrnehmen. Sie verbinden mit muslimischen Patienten eine durch sprachliche Verständigungsprobleme, andersartiges Krankheitsverständnis sowie mit Berührungsängsten belastete Untersuchungs-

${ }^{1}$ http://de.statista.com/statistik/daten/studie/72321/ umfrage/entwicklung-der-anzahl-der-muslime-indeutschland-seit-1945/http://www.bpb.de/nachschlagen/zahlen-und-fakten/soziale-situation-in-deutschland/145148/religionszugehoerigkeit

\section{Abstract}

Introduction: Due to the increasing numbers of Muslims in Germany ${ }^{1}$ - about 4.3 million at the moment - more Muslim patients are medicated in the practices of family doctors. Their heterogeneous cultural and religious backgrounds are nontheless unknown and unfamiliar for the treating general practitioner. Based on the daily experiences of the latter and in order to capture their development of intercultural competence, in the present study a brainwriting with general practitioners was conducted to record their spontaneous associations with Muslim patients. Methodology: Individually and without exchange 90 general practitioners (66 male, 24 female) listed subjective thoughts regarding „Muslim patients" on a prepared sheet of paper. Additionally, sex, age, number of years as physician in a private practice and the frequency of treatment of Muslim patients in their own practice were requested. The content of the notes were evaluated using MAXQDA and were clustered in the categories of "language", "company", "violence", "men”/,women”, "psychosomatic medicine”, "compliance", "understanding of illness", "physical examination" and "head scarf".

Results: The ideas listed show that the majority of interviewed general practitioners regarded the treatment of Muslim patients as difficult. They associate Muslim patients with communication problems, a different type of disease understanding and a fear of contact, which hampers the examination situation. Less frequently, positive associations and unproblematic examination situations were noted.

Conclusions: Due to a lack of knowledge about cultural and religious contexts Muslim patients are often described by using stereotypes. This underlines the necessity to foster intercultural competences and self-reflection in daily practice and its systematic inclusion in medical education. 
situation. Weniger häufig wurden positive Assoziationen und unproblematische Untersuchungssituationen notiert.

Schlussfolgerungen: Aufgrund mangelnden Wissens über kulturelle und religiöse Kontexte werden muslimische Patienten in den Ergebnissen wenig reflektiert und vielfach stereotypisierend dargestellt. Dies verdeutlicht die Notwendigkeit interkulturelle Handlungskompetenz in der Alltagspraxis zu fördern und systematisch in den Ausbildungsprozess einzugliedern.

\section{Einleitung}

„Orientieren Sie die Therapie an den Lebensbedingungen Ihres Patienten“ [1] - eine Forderung der sicherlich alle Hausärzte zustimmen. Im Umgang mit muslimischen Patienten sehen sich nicht-muslimische Hausärzte in Deutschland vor die Herausforderung gestellt, trotz eigener Unwissenheit über die vollständigen, anderen Lebenszusammenhänge eine ganzheitliche Versorgung anzubieten. Etwa 5 Prozent der deutschen Bevölkerung zählen zu der intern sehr heterogenen Gruppe von Muslimen. Ärzte kommen zunehmend häufiger in die Situation, dass ihre Behandlung von Personen dieses Kultur- bzw. Religionskreises, in Anspruch genommen wird.

Vor diesem Hintergrund ist die Ausprägung der Entwicklung der interkulturellen Handlungskompetenz und die Selbstreflexion von Hausärzten von Interesse [2,3]. Der vorliegende Artikel ist das Ergebnis einer ersten Untersuchung zu dieser Thematik mit deutschen ${ }^{2}$ niedergelassenen Hausärzten und stellt spontane Assoziationen zu dem Stichwort „Muslimischer Patient“ dar (siehe $\odot$ Abb. 1).

\section{Methodik}

\section{$\nabla$}

Um einen primären Eindruck über erlebte Situationen mit muslimischen Patienten und gegebenenfalls vorhandenen Stereotypen bei unserer Untersuchungsgruppe zu gewinnen, wurde eine abgewandelte Form des Brainwritings mit deutschen Hausärzten durchgeführt. Durch das anonyme, schriftliche Notieren ermöglicht die Methode eine unbeeinflusste und ungehemmte Erfassung vielfältiger, spontaner Ideen und Assoziationen zu einer festen Fragestellung [4].

Für den Tübinger Tag der Allgemeinmedizin 2010 wurde ein Formblatt vorbereitet und an die 90 anwesenden Hausärzte, die gleichzeitig als Lehrärzte tätig sind, ausgeteilt. Auf diesem Blatt konnte unter der Überschrift „Stichwort- und Gedankensammlung: Muslimischer Patient - was fällt Ihnen dazu ein?" eine Liste persönlicher Gedanken und Erfahrungen zu dieser Thematik notiert werden. Weiterhin wurde Alter, Geschlecht, Jahre der Niederlassung als Hausarztarzt sowie Häufigkeit der Behandlung von muslimischen Patienten in der eigenen Praxis (mit den Ankreuzoptionen „nie“, „selten“, „häufig“) auf dem Papierbogen abgefragt. Alle anwesenden Hausärzte nahmen an dem Brainwriting teil ( $N=90 ; 66$ männlich, 24 weiblich).

Diese Listen wurden eingesammelt und durchnummeriert, die notierten Kommentare digitalisiert und im ersten Schritt mit dem Datenauswertungsprogramm MAXQDA induktiv-thematisch geclustert und typisiert. Insgesamt sind die Stichworte und Kommentare nicht nur aufschlussreich, weil diese die persönliche Einstellung im Praxisalltag bestimmen. Da diese Ärzte auch in der Lehre tätig sind, können sie auch aufzeigen, welches Bild der Patientengruppe möglicherweise an Studierende vermittelt wird.

In der folgenden Ergebnisdarstellung werden hinter den Zitaten jeweils zuerst die Nummer des zitierten Dokuments sowie des darauf zitierten Stichworts angegeben.

In einem zweiten Untersuchungsschritt wurde geprüft, ob es Zusammenhänge mit den ebenfalls erhobenen Daten zur Person und den genannten Stichworten gibt, die notiert wurden. So wurde eine Subgruppenanalyse möglich.

\section{Ergebnisse \\ $\nabla$}

Die befragten Personen waren im Durchschnitt 13 Jahre als Hausarztarzt niedergelassen. Die Hälfte gab einen häufigen Kontakt mit muslimischen Patienten an $(n=48)$, die andere Hälfte einen seltenen $(n=42)$. Die Antworten reichten von einem Stichwort $(\mathrm{n}=3)$ bis hin zu mehreren Sätzen.

Aus den notierten Stichworten und Gedanken $(\mathrm{N}=258)$ der 90 anwesenden Hausärzte ergaben sich die folgenden 9 Themenkomplexe:

- „Sprache“ ( $\mathrm{n}=69$, Aussagen über deutsche Sprachkenntnisse von muslimischen Patienten)

- „Untersuchung“ ( $\mathrm{n}=42$, Beschreibung und Einschätzung der Untersuchungssituation)

- „Kopftuch“ ( $\mathrm{n}=26$, Erwähnung und Beschreibung von der Verschleierung muslimischer Frauen)

${ }^{2}$ Gemäß Staatszugehörigkeit und Geburtsort

\begin{tabular}{|c|c|c|c|c|c|c|c|c|c|c|}
\hline Codesystem & Beglei... & Gewalt & Psychoso... & Compli... & Krankheitsverst... & Untersuc... & Kopft... & Spra... & Män... & Frauen \\
\hline Begleitung & & & 3 & 2 & 7 & 10 & 6 & 17 & 8 & 28 \\
\hline Gewalt & & & 4 & & 1 & & & 3 & 5 & 4 \\
\hline$\odot$ Psychosomatik & 3 & 4 & & 1 & 13 & 6 & 3 & 4 & 6 & 13 \\
\hline Compliance & 2 & & 1 & & 3 & 5 & & 10 & & 5 \\
\hline- Krankheitsverständnie & 7 & 1 & 13 & 3 & & 14 & 7 & 12 & 4 & 18 \\
\hline Untersuchung & 10 & & 6 & 5 & 14 & & 3 & 18 & 10 & 29 \\
\hline$\odot$ Kopftuch & 6 & & 3 & & 7 & 3 & & 9 & 3 & 18 \\
\hline Sprache & 17 & 3 & 4 & 10 & 12 & 18 & 9 & & 10 & 38 \\
\hline Männer & 8 & 5 & 6 & & 4 & 10 & 3 & 10 & & 25 \\
\hline Frauen & 28 & 4 & 13 & 5 & 18 & 29 & 18 & 38 & 25 & \\
\hline
\end{tabular}

Abb. 1 Kategorien der Assoziationen mit muslimischen Patient/innen von Hausärzt/innen und Häufigkeit der Nennungen. 
• „Männer“/„Frauen“ ( $\mathrm{n}=29 / \mathrm{n}=82$, Aussagen zu Geschlechterrollen und geschlechtsspezifischem Verhalten gegenüber dem/der Behandelnden)

- „Begleitung“ ( $\mathrm{n}=30$, die Begleitung von Patienten von Familienangehörigen beim Hausarztbesuch)

- „Gewalt“ ( $\mathrm{n}=7$, aggressives Verhalten im Untersuchungskontext sowie Anzeichen und Berichte über Gewalt in familiären Kontext)

- „Krankheitsverständnis“ ( $\mathrm{n}=37$, Beschreibung des wahrgenommenen Krankheitsverständnisses von muslimischen Patienten sowie die Beschreibung der Beschwerden dem Hausarzt gegenüber)

- „Psychosomatik“ ( $\mathrm{n}=16$, Aussagen über psychosomatische Erkrankungen unter muslimischen Patienten)

- „Compliance“ $(\mathrm{n}=15$, Umsetzung angeordneter medizinischer Therapie).

Am häufigsten wurden Aussagen zu Begleitung, Sprache, Untersuchung, Krankheitsverständnis und Frauen/Verschleierung getätigt, wobei manche Assoziationen mehreren Themenkomplexen zugeordnet, d.h. doppelt kodiert, sind. Die Grafik stellt die Kategorien und die Anzahl der dazugehörigen Notizen dar.

Im Folgenden werden die Ergebnisse dazu einzeln dargestellt, wobei die zitierten Äußerungen der Hausärzte durch kursive Schrift und Anführungszeichen hervorgehoben werden.

\section{Themenkomplex Sprache}

69 Hausärzte thematisierten Verständigungsprobleme aufgrund mangelnder deutscher Sprachkenntnisse seitens der Patienten, insbesondere der Frauen. Das Sprachproblem wird - wie in der Fachliteratur auch [5]- als die Größte und im Vergleich zu kulturell-religiösen Hintergründen als schwerwiegendere Behandlungshürde dargestellt. Dadurch wird die Untersuchungssituation eingeschränkt, was eine unbefriedigende Diagnose- und Adhärenz zur Folge haben kann:

„Muslimisch ist kein Hindernis, sondern nur mangelnde Sprachkenntnisse “ $[10,11]$

„Fazit: Problem nicht die Religion, sondern die Sprachbarriere“ $[42,9]$.

Als Lösungsansätze für dieses verbreitete Problem werden in der Literatur vorrangig speziell ausgebildete Medizindolmetscher vorgeschlagen, da sich Patienten ernst genommener fühlen, wenn sie ihre Beschwerden in der Muttersprache schildern können. Zusätzlich könnte dies die nachgewiesene geringere Informationsvermittlung und affektive Aufmerksamkeit besser kompensieren [6-9]. Ihre Rolle wird differenziert bewertet. Der einfachere und kostengünstigere Einsatz von Familienangehörigen oder Praxis-/Krankenhauspersonal als Dolmetscher ist jedoch ebenfalls medizinisch-ethisch problematisch, da in ersterem Fall aus Intimitätsgründen möglicherweise nicht alles erzählt bzw. übersetzt wird. Bei professionellem Personal aus der gleichen Herkunftsregion besteht zusätzlich das Problem, dass Übersetzungen entsprechend kultureller Normen getätigt werden. Übersetzungsleistungen werden nicht zwangsläufig von den behandelnden Ärzten in Anspruch genommen [10-12].

\section{Themenkomplex Untersuchung}

Neben der Sprache kann die Untersuchungssituation aufgrund der

„Zurückhaltende Distanz des Patient bzgl. körperlicher Untersuchung“ $(74,5)$

sowie eines hohen Schamgefühls als schwierig empfunden werden, was auch beim behandelnden Hausarzt Unsicherheiten aufbaut $(26,8)$. Gemäß medizin-ethischer islamischer Kriterien muss nicht das Geschlecht, sondern die Kompetenz, das primäre Auswahlkriterium für einen Arzt sein. Gleichwohl sollte bei entsprechender Verfügbarkeit vorrangig ein muslimischer, gleichgeschlechtlicher Mediziner gewählt werden [13].

Bei weiblichen Patientinnen erweist sich, laut der Hausärzte, den Hausärzten die Behandlung besonders schwierig wegen geringer Kenntnisse der deutschen Sprache und durch das Tragen eines Kopftuchs und anderer Kleidung. Seitens der Hausärzte kann dies, aus Unklarheit über den richtigen Umgang mit Körperkontakt, Berührungsängste schaffen. Die Fachliteratur verweist weiterhin darauf, dass bei muslimischen Patientinnen, bspw. aufgrund der Kleidergewohnheiten, mehr Zeit für die Untersuchung eingeplant werden sollte [5].

Die befragten Hausärzte äußerten sich doppelt so häufig wie die befragten Hausärztinnen zu Kleidung und 3-mal so häufig zu Kopftuch und mangelnder Autonomie von muslimischen Patientinnen.

\section{Themenkomplex Kopftuch}

Überwiegend wird das Kopftuch als hinderlich für den Untersuchungsvorgang bewertet, was sich bereits bei der Begrüßung abzeichnen kann:

„Verschleierte Frauen, die den Handschlag vermeiden und den männlichen Begleiter als Vermittler nutzen“ [32, 5].

Die Hausärzte, die muslimische Patienten mit Kopftuch verbinden, treffen Aussagen von:

„Wie offen Frauen sind, wie schnell sie sich bei Untersuchungen aus Kopftuch usw. schälen, erstaunt“ [68, 7]

„Keine Burka, häufig ohne Kopftuch“ [57, 6]

bis hin zu diskriminierenden Assoziationen:

„Schleiereulen“ [49, 6].

\section{Themenkomplex Männer/Frauen}

In Bezug auf das Geschlechterverhältnis wird gemäß den Stichworten den männlichen muslimischen Patienten häufig ein rücksichtsloses bis herrisches Verhalten von den Hausärzten attestiert:

„Starke Dominanz der Männer“ [61, 7].

Für Hausärztinnen können daraus auch eine eigene Verunsicherung und ein Empfinden von Missachtung gegenüber ihrer Person resultieren:

„Eher Probleme mit männlichen Patienten, manchmal habe ich das Gefühl sie nehmen mich nicht ernst, weil ich Ärztin bin“ [27, 10]. In der medizinischen Fachliteratur werden unterschiedliche Verhaltensweisen männlicher und weiblicher muslimischer Patienten in der Hausarztpraxis bislang nicht aufgegriffen. Auch unterschiedliche Gefühle der Hausärzte sind bisher nicht thematisiert.

In mehreren notierten Kommentaren werden die Frauen mit Bewegungsmangel und Übergewicht in Verbindung gebracht. Während ihnen überwiegend eine untergeordnete Rolle in der Familie zugeschrieben wird, nehmen 2 Hausärzte gerade junge weibliche muslimische Patientinnen als selbstbewusst bis distanzlos wahr:

„Generation 18-30 Jahre fordernd, freundlich, aber öfters distanzlos, überselbstbewusste Frauen“ [13, 6].

\section{Themenkomplex Begleitung}

Die häufige Begleitung besonders von weiblichen muslimischen Patientinnen bei Untersuchungsterminen wird von knapp einem Drittel der Hausärzte thematisiert: 
„Viele Kinder, dolmetschend“ [81, 12]

„Selten allein, Frauen häufig in Begleitung, meist wird die gesamte Familie behandelt" $[31,8]$.

Speziell bei Frauen wird die Begleitung damit begründet, dass Familienangehörige, z. B. die Kinder oder der Ehemann, als Dolmetscher fungieren. Überwiegend sind die befragten Hausärzte der Meinung, dass muslimische Patienten deutlich häufiger als Patienten mit anderem religiösen oder soziokulturellen Hintergrund ihre Arztbesuche nicht allein durchführen. Dies kommt zustande, weil bei ihnen Entscheidungen häufig im Familienkreis getroffen werden $[7,11,14]$. Die teilnehmenden Hausärzte der Untersuchung bewerten dies tendenziell negativ:

"Treten im Clan auf“ [34, 5].

„Kommen meist zu mehreren, ohne das beim Terminieren zu sagen, sprengt den Rahmen einer normalen Sprechstunde“ $[10,6]$.

\section{Themenkomplex Gewalt}

Über das erwähnte autoritäre Verhalten Frauen gegenüber hinaus verbinden die Hausärzte auch Zwangsheirat und Gewalt im familiären Kontext mit muslimischen Patienten:

„Viele verdeckte Hinweise auf häusliche, evtl. nur verbale, Gewalt“ $[68,10]$.

$\mathrm{Zu}$ muslimischen Patientinnen sind keine gesonderten Zahlen verfügbar, Schröttle zeigt jedoch auf, dass jede dritte bis vierte Frau türkischer Herkunft unter 60 Jahren Opfer körperlicher und/oder sexueller Gewalt wurde [15]. Die Ursachen liegen jedoch stärker in traditionellen Vorstellungen von Ehre, da sich familiäre Gewalt nicht mit dem Koran legitimieren lässt [16]. So ist auch eine Assoziation muslimischer Patienten mit Gewaltbereitschaft gegenüber dem Behandelnden zu verstehen:

„Gewaltpotenzial bei Kränkung, Morddrohung nur aus dieser Klientel“ $[1,11]$.

\section{Themenkomplex Krankheitsverständnis}

Der diagnostische und therapeutische Behandlungsprozess ist weiterhin beeinflusst durch ein häufig weniger naturwissenschaftlich geprägtes Krankheitsverständnis. Aufgrund einer weniger starken Trennung von Körper und Seele wird das Krankheitserleben und -verständnis von muslimischen Patienten stärker in Zusammenhang mit psychosomatischen Erkrankungen gebracht [17]. Kulturspezifische Beschreibungsformen von Beschwerden, die in den Augen westlicher Mediziner wie psychotische Störungen erscheinen, wie bspw. „Wind in den Knochen", sind vielfach aus der Fachliteratur bekannt $[7,14]$. Trotzdem wird diese für westliche Medizinern meist ungewohnte Schmerzbeschreibung fehlinterpretiert und führt zu „diskriminierenden Verlegenheitsdiagnosen“ [14].

Die an der Erhebung teilnehmenden Hausärzte notierten hierzu bspw.:

„Sie haben diffuse, depressive Beschwerden, die konkrete Hilfen erwarten (Spritzen, Antibiotika), welche in der Regel nur kurz helfen“ $[16,7]$.

Diese nehmen die Hausärzte als Folge von psychischen Problemen wahr, die von den Patienten als körperlichen Schmerzen formuliert werden. In dieser Form werden sie in dem persönlichen Umfeld der Patienten leichter sozial akzeptiert:

„Körperliches Leiden ist akzeptiert, psychisches Leiden eher nicht“ [48, 9].

Aus dem gleichen Grund werden aber entsprechende psychotherapeutische Behandlungen schwer angenommen $(8,11,19,31)$. Dies resultiert allerdings auch aus einem Mangel an Wissen über den Umgang mit psychosomatischen Störungen, welches kein migrantenspezifisches Problem darstellt [17].

\section{Themenkomplex Psychosomatik}

Das Zurückweisen von psychotherapeutischen Behandlungen steht eng in Verbindung mit den häufigen Assoziationen der Hausärzte mit Depression und Somatisierung bei muslimischen Patienten:

„Depressionen, deren - familiäre Hintergründe nicht einfühlbar sind“ $[21,11]$.

„Psychischer Leidensdruck äußert sich in körperlichen Beschwerden“ $[48,5]$.

Die Ursache verschiedener körperlicher Leiden sowie Hauptbeschwerden von muslimischen Patienten schreiben die Hausärzte folglich stärker als die Patienten selbst psychisch-sozialen Kontexten zu.

Brucks sowie Wunn und Klein verweisen darauf, dass psychische Probleme wie Depression als eine typische Folge migrationsspezifischer Erlebnisse auftreten können. Viele der muslimischen Patienten in Deutschland haben eine Migrationserfahrung gemacht. Zusätzlich weisen viele muslimische Patienten in Deutschland einen relativ niedrigen sozioökonomischen Status auf, der viele Probleme mitbestimmt [17-19]. Sie müssen in diesen Fällen als solche erkannt und entsprechend von kultur- und religionsspezifischen Problemen getrennt betrachtet werden.

\section{Themenkomplex Compliance}

Aussagen über die Compliance muslimischer Patienten reichen von neutralen über relativierende Darstellungen wie

„Individuell sehr schwankende Compliance“ $[52,8]$

bis zu einer negativen Bewertung aufgrund von Verständigungsproblemen $(38,7)$ und aufgrund des kulturell-religiösen Hintergrundes $(18,5)$ in 6 Fällen. 2 Hausärzte nennen ein geringes Interesse an Vorsorge $(35,14 ; 13,13)$. Demgegenüber steht die Wahrnehmung einer

„Gute[n] Compliance, fast unterwürfig, teils zwanghaft“ [31,5]. Dies verdeutlicht die unterschiedlichen Erfahrungen, die Hausärzte in der Behandlung von muslimischen Patienten machen können [20].

\section{Begrenzung der Studie \\ $\nabla$}

Das Brainwriting erfasst spontane Assoziationen. Trotz teilweise ausführlicher Sätze, lassen teilweise unkommentierte Stichwörter eine breite Interpretation zu. Verallgemeinerungen und Stereotypisierungen in unserem Artikel können darauf zurückzuführen sein.

Nicht abgefragt wurde, wie von den Hausärzten „muslimisch“ jeweils definiert wird. Dies bedeutet, dass sich die notierten $\mathrm{Ge}$ danken sowohl auf streng religiöse wie auch nicht religiöse Patienten, die aus überwiegend islamischen Ländern stammen, beziehen. Für uns liegen damit keine Differenzierungskriterien zwischen religiös bzw. kulturell bedingten Schwierigkeiten (auch aus Sicht der Betroffenen) in der Behandlung vor. Dies ist zu einem Teil dem Untersuchungsdesign geschuldet, welches nicht auf eine explizite Unterteilung angelegt ist. Es wurde auch bewusst mit dem Label „türkisch-muslimisch“ und der Brainwriting Methode gearbeitet. Dadurch kann der Einfluss der gesamtgesellschaftlichen und -politischen Diskussion über „die Muslime" ungefilterter aufgegriffen werden. Die Offenlegung von Stereotypen ermöglicht eine Be- und Verarbeitung für die 
einzelnen Hausärzte bzw. auch Leser des Artikels. Jedoch ist es auch Teil der Ergebnisse selbst, dass weniger als 10\% der Befragten eine Differenzierung vornimmt, bzw. auf die Heterogenität muslimischer Patienten verweist. Dies bietet gute Ansatzpunkte für weitere Untersuchungen.

\section{Diskussion}

$\nabla$

Die notierten Gedanken zu muslimischen Patienten drehen sich überwiegend um die Untersuchungssituation, Krankheitsverständnis, psychische Probleme und Compliance.

In ihrer Darstellung dominiert ein negativer Grundtenor: Aus Sicht der Behandelnden schaffen sprachliche Verständigungsprobleme, eine teilweise ungenügende Beschreibung der Beschwerden sowie Kleidungsvorschriften und starke Schamhaftigkeit unbefriedigende Möglichkeiten für eine adäquate Anamnese und entsprechende Diagnose. Einzelne positive Beschreibungen heben sich davon ab:

„Durchweg gute Erfahrungen“ [71, 6].

Die Breite der Assoziationen, die Hausärzte mit muslimischen Patienten haben, verdeutlicht einerseits die Heterogenität einer nicht zu typologisierenden Patientengruppe. Andererseits zeigt sie die Subjektivität der Wahrnehmung von den behandelnden Hausärzten, was intendiert war. Nur so sind Stereotype erfassbzw. aufdeckbar.

Implizit werden muslimische Patienten in den Ergebnissen als eine homogene Gruppe, die sich von der „normalen Behandlungspraxis“ der Hausärzte sowie anderen Patientengruppen problematisch abhebt, dargestellt. Der individuelle Kontext des jeweiligen Patienten scheint weniger präsent zu sein. Eine unbewertete Relativierung und Differenzierung von muslimischen Patienten wurde nur in wenigen Fällen den Notizen vorangestellt:

„Heterogene Gruppe, daher Stichworte mit Zurückhaltung“ [2, 1] „Breite Spanne von streng bis locker praktiziertem Muslimiszismus“ [57, 1].

Differenziert wird insofern, dass die Probleme im Untersuchungskontext weniger religiösen Aspekten als sprachlichen Problemen oder kulturellen Unterschieden zugeschrieben werden:

„Auch als Arzt spürt man Hilflosigkeit, da sprachliche und kulturelle Barrieren, um auf des Pudels Kern - das eigentliche Problem - des Patienten zu stoßen“ [5,9].

Die eigenen kulturell geprägten Wertvorstellungen der Hausärzte beeinflussen die Einschätzung des Gegenübers sowie das eigene Empfinden in der Arzt-Patienten-Beziehung. Ein geringer Einblick in die Lebenswelt wird vonseiten der Hausärzte nur beiläufig thematisiert $(82,10 ; 21,11)$. Werden dadurch sozioökonomische Probleme nicht als solche erkannt, entsteht die Gefahr einer „Ethnisierung“ bestimmter Verhaltensweisen und Beschwerden [5].

Konstruierte Unterschiede prägen sowohl die Untersuchungssituation und -kommunikation als auch Erwartungshaltungen an Behandlungsinhalte und Medikation (psychosomatische Erkrankungen, eng verbunden mit einer patientenseitigen „Pharmagläubigkeit“). Konstruiert und essenzialisiert ist die Andersartigkeit, wenn die Wahrnehmung besteht, dass muslimische Patienten grundsätzlich andere Bedürfnisse haben und sich ihre diagnostische und therapeutische Behandlung daher von der weiterer Patienten unterscheiden. Dies kann bei der Adhärenz zu Reibungspunkten führen und eine individuell patientenorientierte, gleichberechtigte, empathische Anamnese und Behandlung verhindern.

Die arztseitigen Handlungsbedarfe liegen folglich in einer Wissenserweiterung und Sensibilisierung gegenüber unbekannter Lebenshintergründe sowie einer empathisch-wertoffenen, individuell auf den jeweiligen Patient abgestimmten Patient-ArztBeziehung. Weiterhin bedarf es einer bewussten Auseinandersetzung mit der eigenen Einstellung gegen über „anderen“ Patientenkohorten - und zwar bereits im Studium [21].

Alle Befragten sind gleichzeitig Lehrärzte. Das bedeutet, dass sie ihre subjektiven Erfahrungen und Wahrnehmungen an Mediziner in der Ausbildung weitergeben, was eine besonders hohe Sensibilität und Selbstreflexion über eigene Werte, Normen und Handlungsmuster erforderlich macht. Gleichzeitig ist es notwendig, das Thema Interkulturalität in die medizinische Ausbildung zu integrieren, um Wissenslücken in der Praxisrealität vorzubeugen und eine frühzeitige Vorbereitung zu ermöglichen. Unserem Wissen nach ist eine systematische, obligatorische Einbindung interkultureller Inhalte in Vorlesungen, Seminare und Praxisübungen, bei Weiterbildungen und Studien von Medizinern bisher die Ausnahme und weitgehend auf das Engagement einzelner Lehrärzte/Dozenten zurückzuführen. Die Annahme, dass sich interkulturelle Handlungskompetenz oder die Kompetenz zur Selbstreflexion quasi automatisch durch die Praxiserfahrung ergibt, wird u. a. durch die vorliegende Studie widerlegt. Neben Hintergrundwissen und Erfahrungen mit spezifischen kulturellen Patientengruppen ist ein grundlegendes Bewusstsein sowie generell professioneller Umgang mit Personen und Kontexten außerhalb des eigenen kulturellen Hintergrundes erstrebenswert [21], da sich interkulturelle Kompetenz primär durch eine innere Haltung auszeichnet. Das meint ein inneres Bewusstsein über die eigene kulturelle Prägung, um Kategorisierungen kritisch zu reflektieren und sich empathisch in die Bedürfnisse des Gegenübers einzufühlen [16]. Für den Umgang mit muslimischen wie weiteren Patienten mit Migrationshintergrund bedeutet dies, sie in ihrer kulturellen und religiösen Heterogenität wahrzunehmen, kultursensibel mit ihnen zu kommunizieren sowie Verallgemeinerungen und Stereotypisierungen durch Selbstreflexion und Toleranz vorzubeugen [11].

Weiterhin muss in der Behandlung zwischen kultur-, religionsund migrationsspezifischen Problemen unterschieden werden [19]. Strukturell könnte dies über eine Dolmetschervergütungsregelung durch die Krankenversicherungen unterstützt werden, nicht zuletzt um Kosten zu sparen, die aufgrund von Fehlbehandlungen entstehen, bspw. weil ein Patient vor seinem dolmetschenden Kind nicht alles sagen will oder kann [12]. Insgesamt kann dies einen bedeutsamen Beitrag leisten, um die beschriebene „eigene Hilflosigkeit“ [80, 8] der Hausärzte zu mindern.

\section{Anmerkung \\ $\nabla$}

Zur Förderung von interkultureller Handlungskompetenz in der hausärztlichen Praxis wurde der Arbeitskreis „Interkulturalität und Hausärztliche Versorgung“ gegründet. Bei Interesse wenden Sie sich bitte an die Erstautorin.

Interessenkonflikt: Die Autoren geben an, dass kein Interessenkonflikt besteht. 


\section{Literatur}

1 Gräf U. Orientieren Sie die Therapie an den Lebensbedingungen Ihres Patienten! 2002; Bd. 224, 224: 15

2 Kronenthaler A. Zur Entwicklung interkultureller Handlungskompetenz. Landau: Verlag Empirische Pädagogik; 2008

3 Kronenthaler A. Die Geschichte vom Stuhl und den Milliarden. [Buchverf.]. Bührmann $A D$. et al. Management ohne Grenzen: Grenzüberschreitende Zusammenarbeit erfolgreich gestalten. Wiesbaden: Springer Gabler; 2013

4 Rosenthal T, Wagner E. Organisationsentwicklung und Gesundheitsmanagement im Gesundheitswesen: Grundlagen- Methoden- Fallstudien. s.l.: medhochzwei Verlag; 2004

5 Binder-Fritz C. Migration und Gesundheit im Spiegel der Allgemeinmedizin. [Buchverf.]. Peintinger M. Interkulturell kompetent. Ein Handbuch für Ärztinnen und Ärzte. Wien: Facultas Verlag; 2011

6 Salman R. Dolmetscher im Sozial- und Gesundheitswesen. Zeitschrift für Flüchtlingspolitik in Niedersachsen. 2002; 89/90: 58-74

7 Weiß J. Migranten in der Onkologie: Kulturdolmetscher erforderlich. Dtsch Med Wochenschr 2011; Bd. 136: 92-93

8 Calliess J. Interkulturelle Kompetenz in der Facharztausbildung von Psychiatern in Deutschland: Ergebnisse einer Umfrage. GMS Zeitschrift für Medizinische Ausbildung 2008; Bd. 25, 3: 1-12

9 Menz F. Ärtzliche Gespräche mit Patienten mit geringen Deutschkenntissen. [Buchverf.]. Peintinger M. Interkulturell kompetent. Ein Handbuch für Ärztinnen und Ärzte. Wien: Facultas Verlag; 2011

10 Meddings F, Haith-Cooper M. Culture and Communication in ethically appropriate care. Nursing Ethics 2008; Bd. 15, 1: 52-61

11 Ilkilic I. Muslimische Patienten und medizinethische Konfliktfelder in der allgemeinen Krankenversorgung. [Buchverf.]. Peintinger M. Interkulturell kompetent. Ein Handbuch für Ärztinnen und Ärzte. Wien: s.n.; 2011; 355-369

12 Langer T. Telefondolmetscher in der Kinderklinik - wie hoch ist der Bedarf und wie zufrieden sind Eltern und Kinderärzte mit ihrer Nutzung? 10. Deutscher Kongress für Versorgungsforschung. 2011; German Medical Science GMS Publishing House; 2011; Doc11dkvf211. doi:10.3205/11dkvf211 (12.10.2011)
13 Padela A, del Pozo PR. Muslim patients and cross-gender interactions in medicine: an Islamic bioethical perspective. J Med Ethics 2011; Bd. 37: 40-44

14 Jundt $K$, Friese $K$. Besonderheiten im Umgang mit muslimischen Patientinnen in der gynäkologischen Praxis. Der Gynäkologe 2006; Bd. 39: 899-904

15 Schröttle M. Zwangsverheiratung, Gewalt und bei Beziehungen von Frauen mit und ohne Migrationshintergrund in Deutschland. Differenzierung und Polarisierung. [Buchverf.] Senioren und Jugend Bundesministerium für Familie. Zwangsverheiratung in Deutschland. Baden-Baden: s.n.; 2007; 149-170

16 Laabdallaoui M, Rüschoff I. Umgang mit muslimischen Patienten. Bonn: Psychatrie Verlag; 2012

17 Brucks U. Migration und psychische Erkrankung. psychoneuro. 30 2004; Bd. 4: 228-231

18 Topsever P, Schwantes U, Hermann M. Ausländnische Patienten. [Buchverf.] M. M. Kochen. Allgemeinmedizin und Familienmedizin. Stuttgart: Georg Thieme Verlag; 2006

19 Wunn I, Klein C. Bedürfnisse muslimischer Patienten. [Buchverf.]. Peintinger M. Interkulturell kompetent. Ein Handbuch für Ärztinnen und Ärzte. Wien: s.n.; 2011; 371-388

20 Borde T, David M, Kentenich H. Erwartungen und Zufriedenheit deutscher und türkischsprachiger Patientinnen im Krankenhaus: eine vergleichende Befragung in einer Berliner Frauenklinik. Das Gesundheitswesen: Sozialmedizin, Gesundheits-System-Forschung, Public Health, Öffentlicher Gesundheitsdienst, Medizinischer Dienst 2002; Bd. 64, 8/9: 476-485

21 Hamilton J. Intercultural competence in medical education - essential to acquire, difficult to assess. Medical teacher 2009; Bd. 31: 862-865 